\title{
FIB-4 and APRI scores for predicting severe liver fibrosis in chronic hepatitis HCV patients: a monocentric retrospective study
}

\author{
Roberto Catanzaro ${ }^{1}$, Alice Aleo ${ }^{1}$, Morena Sciuto ${ }^{1}$, Luca Zanoli², Baskar Balakrishnan³ ${ }^{3}$ Francesco Marotta ${ }^{4}$ \\ 'Department of Clinical and Experimental Medicine - Gastroenterology Section, "Gaspare Rodolico" Policlinico Hospital, University of Catania, \\ Catania, Italy \\ 2Department of Clinical and Experimental Medicine, "Gaspare Rodolico" Policlinico Hospital, University of Catania, Catania, Italy \\ ${ }^{3}$ Mayo Clinic, Rochester, MN, USA \\ ${ }^{4}$ ReGenera R\&D International for Aging Intervention, Milano, Italy
}

\begin{abstract}
Aim of the study: Hepatitis C virus (HCV) can cause a chronic liver infection which could then develop into fibrosis, cirrhosis, and hepatocellular carcinoma. Today the diagnosis of liver fibrosis also includes the use of biomarkers. The purpose of our study was to determine the ability of the fibrosis index based on four factors (FIB-4) and aspartate aminotransferase-to-platelet ratio (APRI) to predict the severity of liver fibrosis or cirrhosis.

Material and methods: Medical records of 106 patients with HCV-related liver fibrosis were analyzed. All patients underwent clinical examination, blood tests (complete blood count, total bilirubin, etc.) and transient elastography. FIB-4 and APRI were calculated for each patient.

Results: Twenty-six patients (24.52\%) had F4 fibrosis, 80 patients (75.48\%) had non-F4 fibrosis (FO-F3). There was a statistically significant difference $(p<0.05)$ between non-F4 fibrosis patients and $\mathrm{F} 4$ fibrosis patients in many parameters, including APRI (F4 fibrosis patients had higher values: $2.06 \pm 3.22$ compared to $0.68 \pm 0.76$ of the non-F4 group; $p=0.044$ ) and FIB-4 (F4 fibrosis patients had higher values: $4.84 \pm 4.14$ compared to 2.29 \pm 2.90 of the non-F4 group; $p=0.006$ ). Receiver operating characteristic (ROC) curve analysis for APRI and FIB-4 revealed that the area under the curve (AUC) of FIB-4 was 0.855 (Cl: 0.813-0.936), while the APRI score had an AUC of 0.767 (Cl: $0.79-0.932$ ).

Conclusions: In this study, patients with severe fibrosis or cirrhosis were found to have a higher FIB-4 value than APRI in the context of chronic hepatitis $C$.
\end{abstract}

Key words: liver fibrosis, biomarkers, hepatitis C virus, FIB-4, APRI.

Address for correspondence:

Prof. Roberto Catanzaro, University of Catania, Italy, e-mail: rcatanza@unict.it

\section{Introduction}

Hepatic $\mathrm{C}$ virus (HCV) infection can result in both acute and chronic hepatitis. Its severity can range from a mild disease lasting a few weeks to a serious and permanent disease. Chronic disease has a tendency to evolve towards fibrosis, cirrhosis, and therefore hepatocellular carcinoma (HCC) [1]. In addition to liver damage, HCV infection causes a variety of extra- hepatic manifestations. These range from the presence of clinically insignificant autoantibodies to the development of diseases that affect a variety of organs and tissues such as mixed cryoglobulinemia, purpura, polyarteritis nodosa, porphyria cutanea tarda, lichen planus, autoimmune disorders, lymphoproliferative disorders, etc. [2, 3]. In the year 2015 the World Health Organization (WHO) estimated that about 70 million people infected with HCV had the chronic form of the disease, with a worldwide prevalence of $1 \%[4,5]$. 
Liver fibrosis consists of excessive deposition of extracellular matrix proteins (ECM), including collagen, which results in the destruction of liver architecture. Chronic HCV infection involves the activation of the immune system, which, among other effects, stimulates the proliferation of myofibroblasts and, therefore, increased production of ECM [6]. The development of liver fibrosis and cirrhosis is determined by multiple mechanisms and it can be considered as the scarring process of the liver in response to injury, supported by a continuous pathological process of inflammation, hepatocyte necrosis, and therefore deposition of ECM. The transition into the cirrhotic form of the disease occurs after about 15-20 years of chronic hepatocellular damage [7].

There are several methods available to clinicians to diagnose liver fibrosis. They can be distinguished in invasive and non-invasive approaches. Invasive approaches include liver biopsy. However, this is very expensive and it is not routinely performed in all centers $[8,9]$.

Therefore, over the years, various imaging methods and biomarkers have been tested for the diagnosis of liver fibrosis [10, 11]. Among the most used biomarkers there are the fibrosis index based on four factors (FIB-4) and the aspartate aminotransferase-to-platelet ratio (APRI). These scores are easy to use; the calculations are simple and quick. They are inexpensive: there are no additional costs, because the constitutive FIB-4 [aspartate aminotransferase (AST), alanine aminotransferase (ALT), platelet (PLT) count] and APRI (AST and PLT count) parameters are included in the routine investigation of any liver disease [12].

The aim of this study was to determine whether FIB4 and APRI scores were able to predict severe fibrosis or cirrhosis and which of the two fibrosis scores was more accurate. These patients would be re-evaluated at a later time by postponing or avoiding a liver biopsy.

\section{Material and methods}

This is a retrospective monocentric study; therefore informed consent was not necessary and its management was notified to the local ethics committee. Our research was conducted in accordance with the principles of the 1975 Declaration of Helsinki ( $6^{\text {th }}$ revision). For the study we considered the medical records of
106 patients with HCV related liver disease who were suitable for treatment with HCV direct-acting antivirals (DAA). Their age ranged from 24 to 89 years.

Liver fibrosis or cirrhosis was diagnosed using clinical, laboratory, ultrasonographic, and transient elastography results. Exclusion criteria were: non-HCVrelated liver disease, coinfection with hepatitis B virus (HBV), coinfection with human immunodeficiency virus (HIV), HCC, decompensated liver cirrhosis.

All 106 patients had undergone an accurate evaluation of anamnesis, clinical examination, blood tests [AST, ALT, $\gamma$-glutamyl transferase (GGT), total bilirubin, serum albumin, complete blood count, serum HCVRNA count done by PCR] and transient elastography.

Blood and biochemical parameters were obtained using the common assays currently available. We considered the following normal ranges: for AST and ALT 0-35 IU/l, for GGT 0-38 IU/l, for total bilirubin 0.3-1.2 $\mathrm{mg} / \mathrm{dl}$, for serum albumin 3.5-5.2 $\mathrm{g} / \mathrm{dl}$, for platelet count $130-400 \times 10^{9} / 1$.

Transient elastography was carried out in every patient using FibroScan (Echosens, Paris, France), just before the possible start with DAA therapy. According to Castera's studies on non-invasive diagnosis of liver fibrosis with transient elastography, patients were classified into:

- patients with no fibrosis or mild fibrosis (liver stiffness $>2.5 \mathrm{kPa}$ and $\leq 7 \mathrm{kPa}$, METAVIR score F0-F1);

- patients with significant fibrosis (liver stiffness $>7 \mathrm{kPa}$ and $\leq 9.5 \mathrm{kPa}$, METAVIR score F2);

- patients with severe fibrosis (liver stiffness $>9.5 \mathrm{kPa}$ and $\leq 12.5 \mathrm{kPa}$, METAVIR score F3);

- patients with cirrhosis (liver stiffness $>12.5 \mathrm{kPa}$, METAVIR score F4) [13].

The study population was then divided into 2 subgroups based on liver stiffness measured by FibroScan: cirrhosis subgroup (F4 fibrosis) and those without cirrhosis (non-F4 fibrosis, including F0-F1, F2 and F3).

The FIB-4 and APRI scores were calculated for each patient and the values obtained were rounded to two decimal places. Based on the available data from the scientific literature, a cut-off value of 3.25 for the FIB-4 and 2.0 for the APRI were used to predict who, among patients, had cirrhosis [14-16]. The formulas used to calculate the scores are shown in Figure 1.

$$
\text { APRI }=\frac{\frac{\text { AST level of patient (IU/I) }}{\text { AST (upper limit of normal) (IU/I) }}}{\operatorname{PLT} \text { count }\left(10^{9} / \mathrm{I}\right) \times 100} \times 100
$$$$
\text { FIB-4 }=\frac{\text { age }(\text { years }) \times \text { AST }(I \mathrm{IU} / \mathrm{I})}{\text { PLT count }\left(10^{9} / \mathrm{l}\right) \times \sqrt{\mathrm{ALT}(\mathrm{IU} / \mathrm{l})}}
$$

Fig. 1. Aspartate aminotransferase-to-platelet-ratio index (APRI) and fibrosis index based on four factors (FIB-4). AST - aspartate aminotransferase, PLT - platelets, ALT - alanine aminotransferase 
Table 1. Demographic and laboratory characteristics of the entire population, patient subgroups and the differences between distributions of certain variables among the subgroups

\begin{tabular}{|c|c|c|c|c|}
\hline Variable & The entire study population & Non-F4 fibrosis & F4 fibrosis & $p$-value \\
\hline Male (\%) & $51(48.11 \%)$ & $35(43.75 \%)$ & $16(61.54 \%)$ & 0.120 \\
\hline Female (\%) & $55(51.89 \%)$ & $45(56.25 \%)$ & $10(38.46 \%)$ & \\
\hline Age (years) & $46.24 \pm 15.36$ & $60.35 \pm 10.63$ & $64.54 \pm 13.16$ & 0.156 \\
\hline AST (IU/I) & $50.11 \pm 38.74$ & $42.33 \pm 26.97$ & $74.08 \pm 56.59$ & 0.010 \\
\hline ALT (IU/I) & $59.99 \pm 63.88$ & $52.36 \pm 58.01$ & $83.46 \pm 75.81$ & 0.063 \\
\hline Platelets $\left(\times 10^{9} / \mathrm{l}\right)$ & $184.52 \pm 66.57$ & $202.40 \pm 63.02$ & $129.50 \pm 43.50$ & $<0.001$ \\
\hline GGT (IU/I) & $54.72 \pm 61.10$ & $44.83 \pm 28.22$ & $85.15 \pm 109.03$ & 0.073 \\
\hline Total bilirubin (mg/dl) & $0.73 \pm 0.43$ & $0.71 \pm 0.43$ & $0.77 \pm 0.43$ & 0.544 \\
\hline Serum albumin (g/dl) & $3.90 \pm 0.76$ & $3.95 \pm 0.72$ & $3.77 \pm 0.87$ & 0.350 \\
\hline Glycaemia (mg/dl) & $105.66 \pm 33.89$ & $102.09 \pm 32.47$ & $116.50 \pm 36.61$ & 0.080 \\
\hline Liver stiffness (KPa) & $10.57 \pm 9.12$ & $6.68 \pm 3.36$ & $24.56 \pm 11.56$ & $<0.001$ \\
\hline APRI & $1.01 \pm 1.78$ & $0.68 \pm 0.76$ & $2.06 \pm 3.22$ & 0.044 \\
\hline FIB-4 & $2.91 \pm 3.41$ & $2.29 \pm 2.90$ & $4.84 \pm 4.14$ & 0.006 \\
\hline
\end{tabular}

AST - aspartate aminotransferase, ALT - alanine aminotransferase, GGT - $\gamma$ glutamyl transferase, APRI - AST-to-platelet ratio index, FIB-4 - fibrosis index based on four factors

\section{Statistical analysis}

Continuous variables are presented as the means (standard deviations); categorical variables are presented as percentages. Pearson's correlation coefficient $r$ was calculated. Clinical and hemodynamic variables were compared using the $t$ test for continuous variables and chi-squared test for categorical variables. The area under the receiver operating characteristic (ROC) curve, which ranges from 0.50 (no discrimination) to 1.0 (perfect discrimination), was also calculated. Statistical analysis was performed using NCSS 2007 and PASS 11 software (Gerry Hintze, Kaysville, UT, USA). A two-tailed $p$-value $<0.05$ was considered statistically significant.

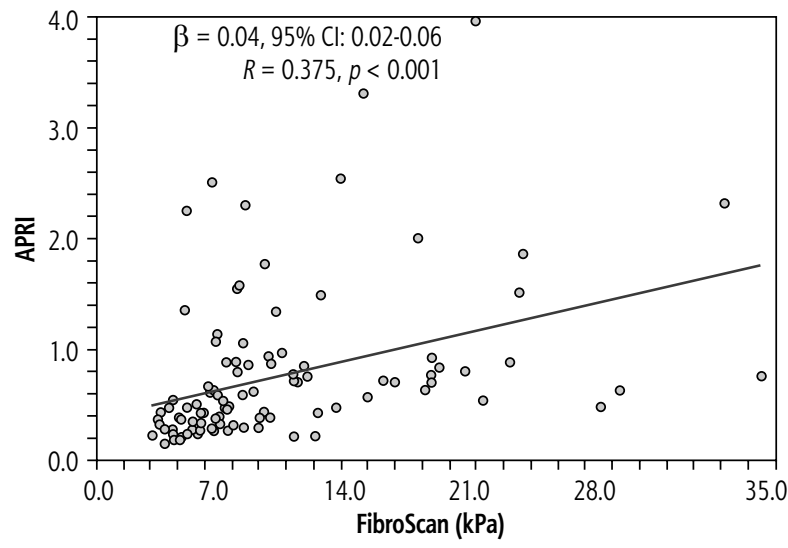

Fig. 2. Association of aspartate aminotransferase-to-platelet-ratio index (APRI) and FibroScan. $\mathrm{kPa}$ - kilopascals

\section{Results}

The demographic and laboratory characteristics of the entire study population and the two sub-populations (those with and those without severe fibrosis) are graphically represented in Table 1.

Twenty-six patients had F4 fibrosis and they represent the $24.52 \%$ of the total; 80 patients had non-F4 fibrosis (F0-F3) and they represent $75.48 \%$ of the total. There were 49 patients with F0-F1 fibrosis and they represent $46.23 \%$ of the total; there were 18 and 13 patients with F2 and F3 fibrosis and they represent $16.98 \%$ and $12.26 \%$, respectively.

Table 1 reports the demographic and laboratory characteristics of all patients, the patient subgroups, and the differences between the distributions of some

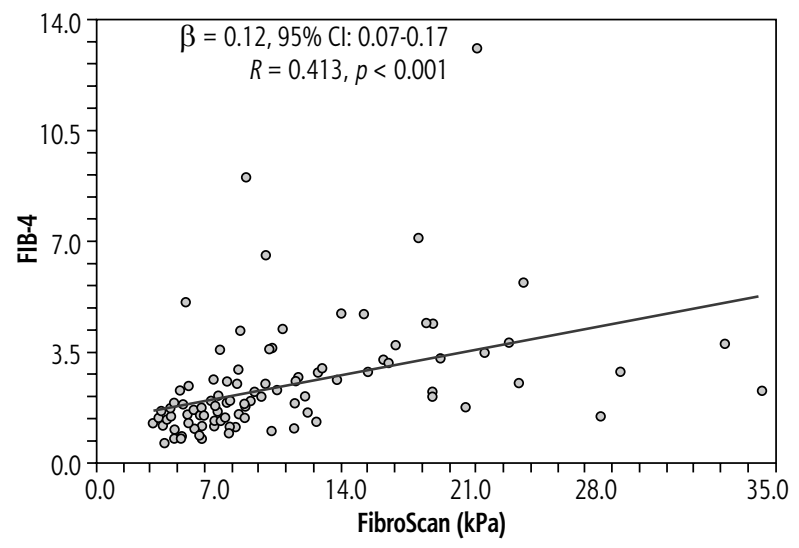

Fig. 3. Association of fibrosis index based on four factors (FIB-4) and FibroScan. $\mathrm{kPa}$ - kilopascals 


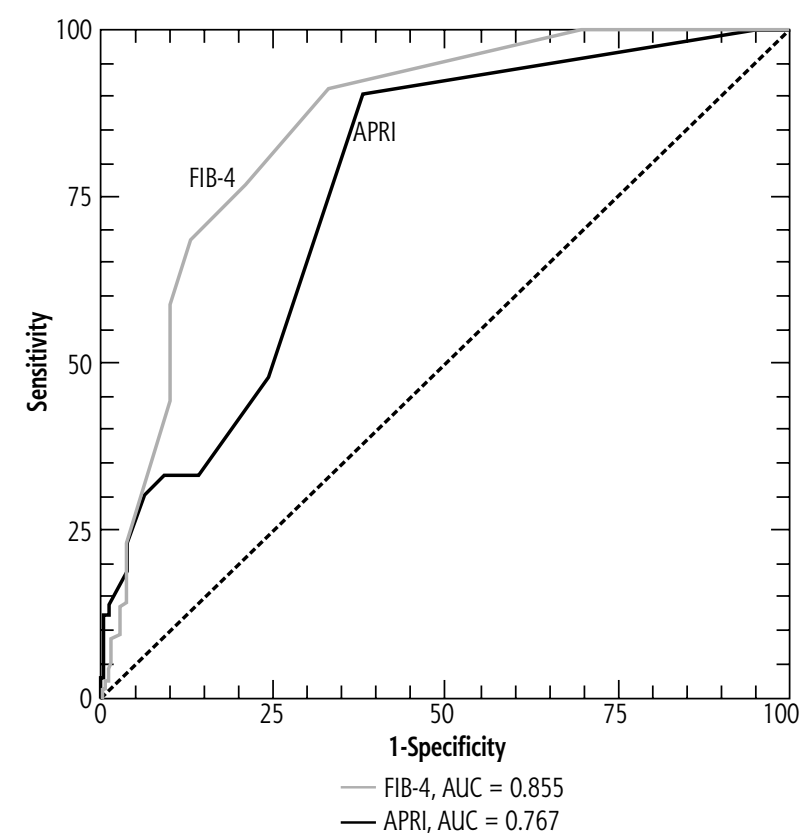

Fig. 4. Receiver operating characteristic curves demonstrating the ability of aspartate aminotransferase-to-platelet-ratio index (APRI) and fibrosis index based on four factors (FIB-4) to predict the presence of severe fibrosis or cirrhosis

variables between the subgroups. There was a statistically significant difference $(p<0.05)$ between patients with non-F4 fibrosis and patients with F4 fibrosis in many parameters. In particular, patients with F4 fibrosis had higher values of AST $(p=0.01)$, APRI $(p=0.044)$, and FIB-4 $(p=0.006)$. However, the platelet levels in these patients were lower $(p<0.001)$. There was not a statistically significant difference $(p>0.05)$ between patients with non-F4 fibrosis and patients with F4 fibrosis in many parameters, such as sex, age, ALT, GGT, etc.

The association of APRI and FIB-4 scores with the stages of fibrosis determined by FibroScan is shown in Figures 2 and 3. Significantly higher APRI and FIB-4 index values were observed among patients with F4 fibrosis.

ROC curve analysis for APRI and FIB-4 scores as predictors of cirrhosis revealed that the FIB- 4 had an area under the curve (AUC) of 0.855 (CI: 0.813-0.936) while the APRI score had an AUC of 0.767 (CI: 0.790.932). The ROC analysis demonstrated that the FIB-4 and APRI scores were indeed able to predict cirrhosis satisfactorily (AUC $=0.875, p<0.001$ for FIB-4 and $\mathrm{AUC}=0.076, p<0.001$ for APRI) (Fig. 4).

\section{Discussion}

As mentioned before, liver fibrosis can develop into more serious and potentially fatal pathological condi- tions [1]. Therefore an early diagnosis is important, so as to prevent or slow down the evolution of fibrosis. Even today, liver biopsy is the method that allows us to make a diagnosis of liver fibrosis with certainty, as it is based on the histological examination [17]. However, it cannot be exploited in daily clinical practice, so over the years we have tried to exploit more of the non-invasive methods. These include transient elastography, which was used to identify the presence and degree of liver fibrosis in the patients of our study. It is an easy and non-invasive method for measuring the stiffness of the liver through use of elastic waves and low frequency ultrasound $(50 \mathrm{~Hz})$. The propagation speed of ultrasound is directly proportional to stiffness: with increasing tissue hardness, the propagation speed of elastic waves increases. Thus, a high result generally indicates the presence of significant liver fibrosis. The final value corresponds to the median of all valid acquisitions, which is considered representative of liver stiffness. This value is expressed in kilopascals $(\mathrm{kPa})$, in a range between 2.5 and $75.0 \mathrm{kPa}$ [8]. However, even in the case of transient elastography there are limits. In fact, false values can occur in the case of obesity, ascites, and restricted intercostal space [18]. Furthermore, false positives can occur during acute viral hepatitis or extrahepatic cholestasis $[19,20]$. Moreover, not all hepatological centers have the FibroScan. Therefore, several serum biomarkers able to identify liver fibrosis have been studied over the years. These serum biomarkers can be distinguished into direct and indirect. Direct ones reflect structural changes in the ECM, including indicators of ECM turnover, fibrogenesis and fibrolysis. Indirect biomarkers are expression of liver damage and/or decline in liver function during the development of fibrosis and cirrhosis. These include routine tests combined with other laboratory or clinical parameters and they comprise ALT, AST, GGT, bilirubin, haptoglobin, apolipoprotein $A_{1}$, and $\alpha 2$-macroglobulin $[14,21]$. APRI and FIB-4 are two of the many scores that can be obtained by combining the various markers, and they have the purpose to increase the diagnostic performance of the markers themselves.

APRI is one of the most studied serum fibrosis indicators in the case of chronic HCV infection. Its calculation derives from the worsening of fibrosis and the portal hypertension that in these patients are associated with the reduction of thrombopoietin production by the hepatocytes, the increase of platelet sequestration by the spleen, and the reduction of AST clearance $[15,21]$.

FIB-4 is also a non-invasive method to assess liver fibrosis. It is based on simple variables (age, AST, ALT and platelet count). It was initially used by AIDS 
researchers of the Pegasys Ribavirin International Coinfection Trial (APRICOT study) to evaluate the presence of liver fibrosis in patients with $\mathrm{HIV} / \mathrm{HCV}$ co-infection [22]. Then, various other studies showed that FIB-4 had a variable degree of accuracy in HCV infected subjects [23-28].

The results of our study are in line with those obtained by other authors. In accordance with the 2018 WHO guidelines, we considered the value of 2.0 for APRI and 3.25 for FIB-4 as cut-off points for cirrhosis [16]. In patients of the F4 fibrosis group, higher values of APRI and FIB-4 were detected than in the non-F4 fibrosis group. From the analysis of the ROC curve an AUC of FIB-4 of 0.855 (CI: 0.813-0.936) was obtained, while APRI score had an AUC of 0.767 (CI: 0.79-0.932). For both markers these results are statistically significant $(p<0.001)$. However, the observation of the two AUC shows that FIB-4 is a better predictor of severe hepatic fibrosis than APRI. This can be explained by the differences in the laboratory parameters taken into account. In fact, the APRI score depends on the AST value and the platelet count (Fig. 1). Comparing the AST value between the two groups of patients, a significant difference $(p=0.01)$ is observed. In fact, AST is more increased in F4 fibrosis patients than in non-F4 patients $(74.08 \pm 56.59 \mathrm{IU} / \mathrm{ml}$ vs. $42.33 \pm 26.97 \mathrm{IU} / \mathrm{ml})$. In contrast, the platelet count is reduced in the F4 fibrosis group compared to the other group $\left(129.50 \pm 43.50 \times 10^{9} / 1\right.$ vs. $202.40 \pm 63.02 \times 10^{9} / 1$; $p<0.001)$. On the other hand, FIB-4 depends not only on AST and platelet count, but also on ALT and age. Neither ALT nor age showed significant differences between the two groups ( $p=0.063$ and $p=0.156$, respectively).

In a study conducted on 575 patients, Papadopoulos et al. obtained results similar to ours. In this study, both APRI and FIB-4 proved effective in predicting significant fibrosis [24]. Papaluca et al. conducted a recent retrospective study based on a cohort of 1007 HCV-positive patients. The authors demonstrated that the use of APRI and FIB-4 scores is a sensitive and reliable non-invasive diagnostic tool and they are good markers to exclude the presence of liver cirrhosis. Furthermore, both APRI and FIB-4 scores reduce the need for transient elastography [29]. In the study of Yen et al. FIB-4 proved better than APRI in the evaluation of liver fibrosis in HCV patients. In fact, in patients with F4 fibrosis, the AUC of FIB- 4 was 0.73 vs. the AUC of APRI, which was 0.70 [26].

The limitations of our study are the small number of medical records analyzed and the retrospective nature of the study. However, we believe that what our data have shown can represent a valid contribution to encourage the use of a non-invasive and certainly reliable method. In the near future we would like to increase this number or even carry out a prospective study with a large sample of patients.

\section{Conclusions}

In conclusion, our study confirms that FIB- 4 and APRI scores are both able to predict severe fibrosis and cirrhosis. FIB-4 was superior to APRI in making a distinction between patients with and without cirrhosis in the setting of chronic HCV infection. Since transient elastography is not readily available in low-income countries, FIB-4 may prove very useful in identifying patients without advanced liver disease in which a liver biopsy could be deferred or avoided.

\section{Disclosure}

The authors declare no conflict of interest.

\section{References}

1. Goto K, Roca Suarez AA, Wrensch F, et al. Hepatitis C virus and hepatocellular carcinoma: when the host loses its grip. Int J Mol Sci 2020; 21: E3057.

2. Kchir H, Kaffel D, Cherif D, et al. Rheumatologic manifestations during chronic viral hepatitis C. Tunis Med 2019; 97: 1251-1257.

3. Gill K, Ghazinian H, Manch R, et al. Hepatitis C virus as a systemic disease: reaching beyond the liver. Hepatol Int 2016; 10: 415-423.

4. WHO. Guidelines for the care and treatment of persons diagnosed with chronic hepatitis $\mathrm{C}$ virus infection. Geneva, Switzerland 2018.

5. Flisiak R, Zarębska-Michaluk D. Perspectives of hepatitis C virus (HCV) elimination in Poland. Clin Exp Hepatol 2019; 5: 210-214.

6. Roehlen N, Crouchet E, Baumert TF. Liver fibrosis: mechanistic concepts and therapeutic perspectives. Cells 2020; 9: 875.

7. Böttcher K, Pinzani M. Pathophysiology of liver fibrosis and the methodological barriers to the development of anti-fibrogenic agents. Adv Drug Deliv Rev 2017; 121: 3-8.

8. Sebastiani G, Gkouvatsos K, Pantopoulos K. Chronic hepatitis C and liver fibrosis. World J Gastroenterol 2014; 20: 11033-11053.

9. Gudowska M, Wrona A, Gruszewska E, et al. Simple non-invasive markers for early diagnosis and determination of the severity of liver diseases. Clin Exp Hepatol 2016; 2: 149-154.

10. Catanzaro R, Sapienza C, Milazzo M, et al. Liver fibrosis: evaluation with diffusion-weighted magnetic resonance imaging in patients with chronic liver disease. Minerva Gastroenterol Dietol 2013; 59: 313-320.

11. Catanzaro R, Milazzo M, Arona S, et al. Diagnostic accuracy of enhanced liver fibrosis test to assess liver fibrosis in patients with chronic hepatitis C. Hepatobiliary Pancreat Dis Int 2013; 12: 500-507.

12. Agbim U, Asrani SK. Non-invasive assessment of liver fibrosis and prognosis: an update on serum and elastography markers. Expert Rev Gastroenterol Hepatol 2019; 13: 361-374. 
13. Castera L, Forns X, Alberti A. Non-invasive evaluation of liver fibrosis using transient elastography. J Hepatol 2008; 48: 835847.

14. Baranova A, Lal P, Birerdinc A, et al. Non-invasive markers for hepatic fibrosis. BMC Gastroenterol 2011; 11: 91.

15. Wai CT, Greenson JK, Fontana RJ, et al. A simple noninvasive index can predict both significant fibrosis and cirrhosis in patients with chronic hepatitis C. Hepatology 2003; 38: 518-526.

16. WHO Guidelines for the care and treatment of persons diagnosed with chronic hepatitis C virus infection. (July 2018) Available at: https://www.who.int/publications/i/item/guide-care-treat-persons-diagnosed-chronic-hepatitis-c-978-92-4-155034-5

17. Zeybel M, İdilman R. Serum biomarkers for the evaluation of liver fibrosis: the need for better tests. Turk J Gastroenterol 2018; 29: 377-378.

18. Sebastiani G, Alberti A. How far is noninvasive assessment of liver fibrosis from replacing liver biopsy in hepatitis C? J Viral Hepat 2012; 19: 18-32.

19. Arena U, Vizzutti F, Corti G, et al. Acute viral hepatitis increases liver stiffness values measured by transient elastography. Hepatology 2008; 47: 380-384.

20. Millonig G, Reimann FM, Friedrich S, et al. Extrahepatic cholestasis increases liver stiffness (FibroScan) irrespective of fibrosis. Hepatology 2008; 48: 1718-1723.

21. Lurie Y, Webb M, Cytter-Kuint R, et al. Non-invasive diagnosis of liver fibrosis and cirrhosis. World J Gastroenterol 2015; 21 : 11567-11583.

22. Sterling RK, Lissen E, Clumeck N, et al. Development of a simple noninvasive index to predict significant fibrosis in patients with HIV/HCV coinfection. Hepatology 2006; 43: 1317-1325.

23. Vallet-Pichard A, Mallet V, Nalpas B, et al. FIB-4: an inexpensive and accurate marker of fibrosis in HCV infection, comparison with liver biopsy and fibrotest. Hepatology 2007; 46: 32-36.

24. Papadopoulos N, Vasileiadi S, Papavdi M, et al. Liver fibrosis staging with combination of APRI and FIB-4 scoring systems in chronic hepatitis $\mathrm{C}$ as an alternative to transient elastography. Ann Gastroenterol 2019; 32: 498-503.

25. Güzelbulut F, Akkan Çetınkaya Z, Sezikli M, et al. AST-platelet Ratio Index, Forns Index and FIB-4 in the prediction of significant fibrosis and cirrhosis in patients with chronic hepatitis $\mathrm{C}$. Turk J Gastroenterol 2011; 22: 279-285.

26. Yen YH, Kuo FY, Kee KM, et al. APRI and FIB-4 in the evaluation of liver fibrosis in chronic hepatitis $\mathrm{C}$ patients stratified by AST level. PLoS One 2018; 13: e0199760.

27. Sripongpun P, Tangkijvanich P, Chotiyaputta W, et al. Evaluation of Aspartate Aminotransferase to Platelet Ratio Index and Fibrosis 4 scores for hepatic fibrosis assessment compared with transient elastography in chronic hepatitis $\mathrm{C}$ patients. JGH Open 2019; 4: 69-74.

28. Yunihastuti E, Wicaksana B, Wiraguna A, et al. Diagnostic performance of APRI and FIB-4 for confirming cirrhosis in Indonesian HIV/HCV co-infected patients. BMC Infect Dis 2020; 20: 372 .

29. Papaluca T, Craigie A, McDonald L, et al. Non-invasive fibrosis algorithms are clinically useful for excluding cirrhosis in prisoners living with hepatitis C. PLoS One 2020; 15: e0242101. 